\title{
Endovascular therapy for chronic cerebrospinal venous insufficiency in multiple sclerosis
}

\author{
Marc A. Lazzaro ${ }^{1 *}$, Osama O. Zaidat ${ }^{1,2,3}$, Nils Mueller-Kronast ${ }^{4 *}$, Muhammad A. Taqii and Douglas Woo ${ }^{1 *}$ \\ ${ }^{1}$ Department of Neurology, Froedtert Hospital and Medical College of Wisconsin, Milwaukee, WI, USA \\ ${ }^{2}$ Department of Neurosurgery, Froedtert Hospital and Medical College of Wisconsin, Milwaukee, WI, USA \\ ${ }^{3}$ Department of Radiology, Froedtert Hospital and Medical College of Wisconsin, Milwaukee, WI, USA \\ ${ }^{4}$ Department of Neurology, Delray Medical Center, Delray Beach, FL, USA
}

Edited by:

Randall Edgell, Saint Louis University, USA

Reviewed by:

Ashish Nanda, University of Missouri, USA

Edgard Pereira, JFK Medical Center, USA

\section{*Correspondence}

Marc A. Lazzaro and Douglas Woo, Department of Neurology, Froedtert Hospital and Medical College of

Wisconsin, 9200 West Wisconsin

Avenue, Milwaukee, WI 53226, USA

e-mail:mlazzaro2@gmail.com;

dwoo@mcw.edu;

Nils Mueller-Kronast, Department of

Neurology, Delray Medical Center,

5162 Linton Boulevard 106, Delray

Beach, FL 33484, USA.

e-mail:muellerkronast@gmail.com
Recent reports have emerged suggesting that multiple sclerosis (MS) may be due to abnormal venous outflow from the central nervous system, termed chronic cerebrospinal venous insufficiency (CCSVI). These reports have generated strong interest and controversy over the prospect of a treatable cause of this chronic debilitating disease. This review aims to describe the proposed association between CCSVI and MS, summarize the current data, and discuss the role of endovascular therapy and the need for rigorous randomized clinical trials to evaluate this association and treatment.

Keywords: angioplasty, stent, jugular, azygos, stenosis, CCSVI, MS

\section{INTRODUCTION}

Multiple sclerosis (MS) is a neurodegenerative disorder that affects approximately 350,000 people in the United States and possibly up to 2.5 million people worldwide (Anderson et al., 1992), making it the most common non-traumatic cause of disability among young and middle-aged people in the developed world (KochHenriksen and Sorensen, 2010). The pathogenesis is complex with varying associations and is primarily thought to be autoimmune, but a unifying hypothesis has yet to be identified. Compelling reports have recently emerged proposing a link between a recently defined entity named "chronic cerebrospinal venous insufficiency" (CCSVI) and MS.

\section{THE CEREBROSPINAL VENOUS SYSTEM IN RELATION TO CCSVI}

A brief overview of the cerebrospinal venous system anatomy and physiology is important to understand the potential role of a vascular model in the pathogenesis of MS.

\section{ANATOMY}

The cerebrospinal venous system is a large interconnected network of venous drainage that spans the length of the neuraxis (Figure 1). The primary intracranial venous drainage can be simplistically divided into deep and superficial systems, while additional drainage occurs through emissary veins. The deep system involves tributaries to the vein of Galen from the internal cerebral veins and the basal veins of Rosenthal. The vein of Galen in turn drains into the straight sinus, confluence of sinuses, and into the transverse sinuses. The superficial system provides cortical drainage into the superior sagittal sinus as well as several large channels that provide lateral cortical drainage. The cortical drainage ultimately passes into the dural sinuses before leaving the head through the transverse sinuses. The two systems communicate by shared outflow through the transverse sinuses.

From the transverse sinuses, two outflow systems provide the majority of venous drainage, the internal jugular veins (IJV) anteriorly, and the vertebral venous system posteriorly (San Millan Ruiz et al., 2002). Each of the paired IJVs receives venous outflow from the transverse sinus, as well as superior and inferior petrosal sinuses, and terminates at the brachiocephalic vein. The vertebral venous system includes the internal and external vertebral venous plexuses. The cervical vertebral veins receive drainage from the internal vertebral venous plexus, condylar veins, and emissary veins (Braun and Tournade, 1977). The vertebral vein forms a plexus around the vertebral artery and courses inferiorly and forms a trunk before draining into the brachiocephalic vein. Communication is present between the vertebral venous system and the azygos vein via the epidural venous plexus and intercostal vein communication (Ibukuro et al., 2001).

The first set of valves encountered in the course of craniocervical venous drainage is at the junction with the brachiocephalic veins. A valve is present in the IJV at the junction with the 


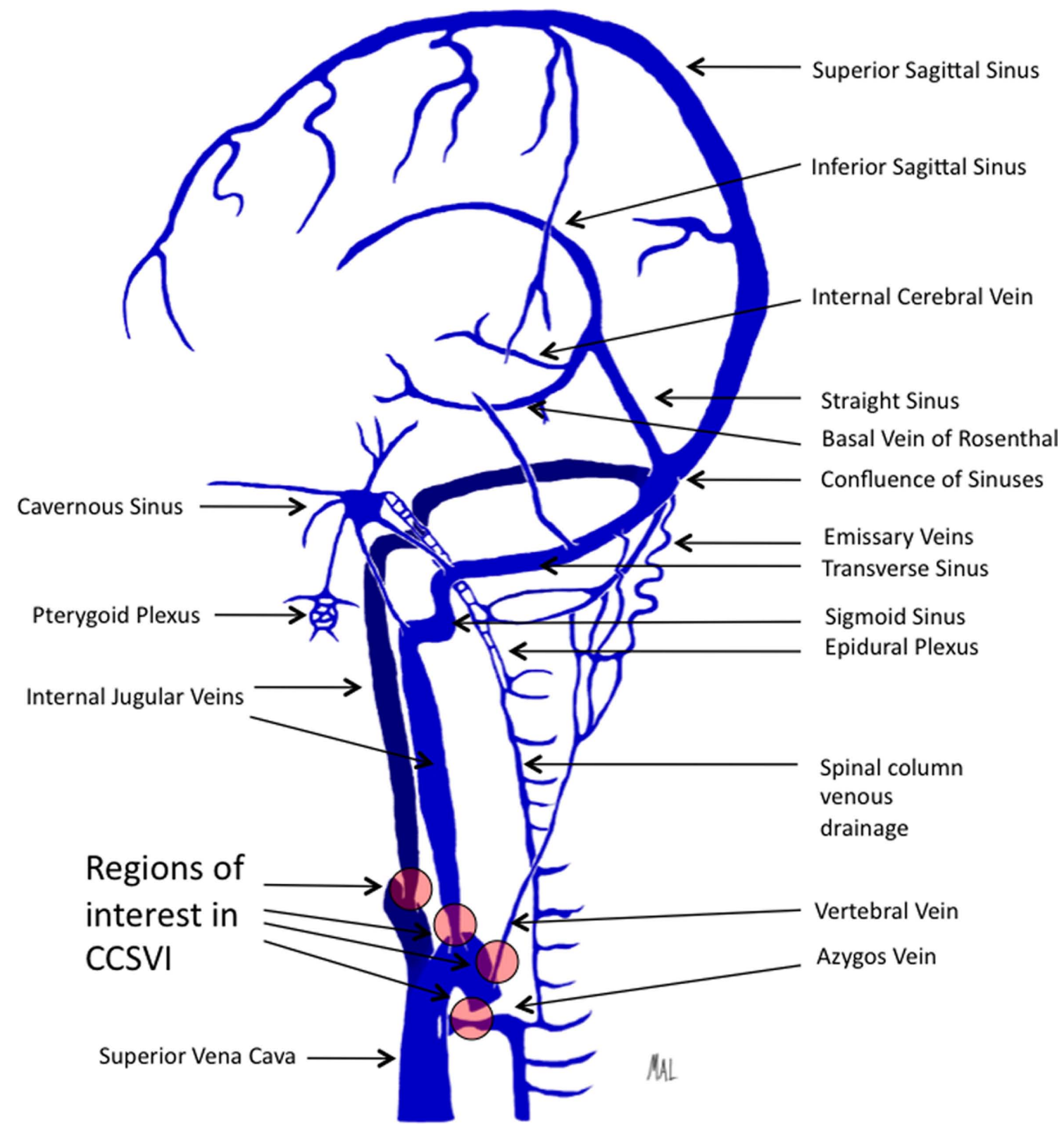

FIGURE 1 | Illustration depicting the predominant veins and sinuses involved in the craniocervical venous outflow. Venous narrowing is depicted at locations of interest in chronic cerebrospinal venous insufficiency.

subclavian vein. This valve may be bicuspid or tricuspid and is commonly located approximately $2 \mathrm{~cm}$ above the junction with the brachiocephalic vein (Sanchez-Hanke et al., 2000). A study of the IJV valve in cadaveric and live human subjects demonstrated the same valve location, mostly bicuspid, and also reported that only $7 \%$ of valves were incompetent out of 41 valves tested (Silva et al., 2002). A similar bicuspid valve is present at the junction of the vertebral vein with the brachiocephalic vein on each side (Chou et al., 2002).

Spinal venous drainage is characterized by a similar large continuous valveless system of veins with comparable complexity and redundancy as is found in the cerebral venous system. The spinal venous drainage shares the cervical outflow with the cranial venous system. Throughout the spinal cord, intrinsic veins drain the parenchyma in a radial pattern into two longitudinal venous trunks, the anterior and posterior median spinal veins (Hurst, 2008). These superficial veins course along the pia mater with segmental drainage via radicular veins to the internal vertebral plexus, or epidural plexus. This multichannel network surrounds the spinal cord in a sleeve-like distribution and extends from the skull base to the sacrum. An external venous system consists of an anterior and posterior plexus that courses along the anterior and 
posterior aspects of the vertebral bodies, respectively. The external system anastomoses with the internal system via intervertebral veins. The spinal venous system drains through deep cervical and vertebral veins at the cervical level and through intercostal and segmental veins into the azygos and hemiazygos veins which drain into the superior vena cava.

The azygos arch courses anteriorly at the level of the fourth and fifth thoracic vertebral bodies to drain into the superior vena cava. A valve is commonly present in the azygos arch, located within $4 \mathrm{~cm}$ of the junction with the superior vena cava, and present in about $65-70 \%$ of cases studied by computerized tomography, which is likely an underestimate due to the study design (Yeh et al., 2004; Steinke and Moghaddam, 2009). In one study, contrast material was visualized posterior to the azygos valve in 53\% of cases suggesting frequent valve insufficiency, but the study was not designed to evaluate competence (Steinke and Moghaddam, 2009).

Embryological development of the venous system involves various stages of growth and invagination that ultimately leads to the complex and variable network forming the cerebrospinal venous system. This intricate series of events leads to variability and can also lead to abnormalities such as focal regions of webbing, stenosis, and valve malformation leading to venous outflow obstruction (Lee et al., 2010).

\section{PHYSIOLOGY}

The cerebrospinal venous system is a large and complex valveless system governed largely by distensibility and intracranial pressure as well as respiratory pressure changes which together lead to antegrade flow (Eckenhoff, 1970). Most studies of venous blood flow are performed with the patient in a supine position which will often demonstrate preferential IJV outflow, however a postural dependence of cerebral venous outflow has also been described (Valdueza et al., 2000). Encephalic venous drainage predominantly exits through the jugular system in the supine position, in contrast to the upright position where blood flow preferentially exits through the vertebral venous system. This is important because most physiologic studies are performed with the subject in the supine position thereby limiting visualization of the vertebral venous drainage system. Corrosion cast studies of the vertebral venous system demonstrate posterior fossa dural venous sinus connections with the vertebral venous system through lateral, posterior, and anterior condylar veins and mastoid and occipital emissary veins (San Millan Ruiz et al., 2002). These findings suggest that complete evaluation of venous outflow in patients may demand more rigorous study including changing postural position. The hydrodynamics of cerebrospinal venous drainage are complex, relying on interplay between arterial blood flow, intracranial pressure, venous system architecture, and intrathoracic pressure as dictated by respiration.

\section{CCSVI IN MS: PROPOSED ASSOCIATION}

The complex pathogenesis of MS partially involves perivenular regions of inflammatory demyelination which are thought to arise from an autoimmune process. The trigger for the autoimmune attack has been unclear, and the mechanism of blood brain barrier (BBB) disruption remains poorly understood, although chronic inflammatory damage promoting permeability and erythrocyte passage has been considered. The vascular theory attempts to answer these questions.

The hypothesis that venous obstruction plays an important role in the development of sclerotic plaques of MS was reported as early as the 1930s. A canine model of cerebral venule obstruction demonstrated histological findings of myelin destruction with axonal preservation and localized proliferation of the fibrous glia (Putnam, 1935). The mechanism of venule obstruction in MS was thought to be due to thrombosis, and this theory was supported by the notion that acute infection and pregnancy alter coagulability and thereby explain the association of these conditions with MS exacerbations.

The vascular model for MS has evolved to the recent theory that chronic venous reflux causes an increase in trans-mural pressure in the microcirculation leading to separation of the tight junctions that form the BBB. Dysfunction of the BBB may then allow erythrocyte and inflammatory cell influx into the extracellular space (Zamboni et al., 2007). These changes could contribute to neurodegeneration by an inflammatory response, which may be exacerbated by venous hypertension induced upregulation of $\mathrm{BBB}$ adhesion molecules, facilitating macrophage and T-cell adhesion, migration, and infiltration, and ultimately leading to iron deposition. Alternatively, the BBB incompetence may not lead to cell migration, but other plasma components such as colloids that would result in osmotic changes leading to cell injury and an inflammatory response (Talbert, 2008).

A recent study investigated the association between abnormalities of the cerebral venous outflow and clinically defined MS (Zamboni et al., 2009a). This study included 65 patients with MS and 235 healthy subjects. All patients underwent screening with transcranial color-coded Doppler sonography and high-resolution echocolor Doppler (TCCS-ECD). Those patients identified to have two or more of five previously defined venous outflow abnormalities (Table 1) subsequently underwent catheter venography. Venography showed no abnormalities in control patients, however those patients with MS had multiple areas of venous stenoses and four distinct patterns of central nervous system venous outflow rerouting (Table 2). Additionally, those patients with relapsingremitting and secondary progressive MS showed venous outflow rerouting patterns significantly different from those with primary progressive MS.

Table 1| Proposed parameters for anomalous venous outflow (Zamboni et al., 2009a).

Presence of two of five TCCS-ECD parameters meets screening criteria for anomalous venous outflow.

Reflux in the IJVs and/or WV in sitting and supine posture

Reflux in the DCVs

High-resolution B-mode evidence of IJV stenosis

Flow not Doppler-detectable in the IJVs and or VVs

Reverted postural control of the main cerebral venous outflow pathways

TCCS, transcranial color-coded Doppler sonography; ECD, high-resolution echocolor Doppler; IJV, internal jugular vein; W, vertebral vein; DCV, deep cerebral vein. 


\section{Table 2 | Four proposed principle patterns of venous rerouting in CCSVI in MS (Zamboni et al., 2009a).}

\section{Proposed venous rerouting patterns in CCSVI}

Type A Steno-obstruction of the proximal azygous with a closed stenosis of one of the IJVs

Type B Significant stenosis of both IJVs and the proximal azygous

Type C Stenosis in both IJVs with a normal azygous system

Type D Azygous system affected in various segments

The vascular model for MS and several compelling reports have generated considerable enthusiasm for investigation of the role of venous obstruction in the pathogenesis of MS. Furthermore, interest has emerged for endovascular therapies including angioplasty and stent implantation for venous stenosis. However, rigorous data demonstrating safety and efficacy are lacking and considerable controversy remains.

\section{FINDINGS SUPPORTIVE OF A LINK BETWEEN CCSVI AND MS}

A canine model of venous obstruction was developed in the 1930s by embolizing part of the longitudinal sinus and adjacent cortical veins (Putnam, 1935). Fourteen dogs survived for varying times that ranged up to 1 year. Histology demonstrated later stages of the lesions having plaques of demyelination with axonal preservation and local dense fibrous gliosis. These findings led the author to conclude that venular obstruction is the immediate antecedent to the formation of typical sclerotic plaques.

Early studies of MS plaques in humans suggested a venocentric pattern of focal inflammatory demyelination, a pattern attributed to an autoimmune process (Fog, 1965; Barnett and Sutton, 2006). Plaque venocentricity has also been demonstrated radiographically using high-resolution magnetic resonance venography (MRV; Tan et al., 2000). Among 95 MS lesions studied, all but one demonstrated a perivenous distribution.

Iron accumulation at sites of MS plaques has been recognized and attributed to the presence of iron-rich macrophages. Iron accumulation may be self-perpetuating by leading to venous endothelial damage and inflammatory response at sites of deposition. In an autopsy study, the incidence of hemosiderin deposition was $30 \%$ in brains of patients with MS, in contrast to only $6 \%$ in brains of normal control patients (Adams, 1988). Furthermore, iron deposition was restricted to regions of plaques in MS patients compared to diffuse deposition seen in normal control brains. Although iron accumulation may result from chronic inflammation and hemosiderin extravasation through a dysfunctional BBB, the severity of venule injury has been considered greater than would be expected from chronic inflammation (Adams, 1988). The vascular model proposes that venous congestion leads to $\mathrm{BBB}$ incompetence and perivenule iron deposition. This may be due to hemosiderin accumulation from microhemorrhages. A recent study of susceptibility weighted imaging found that 12 of 14 MS patients imaged had evidence of increased iron deposition in the medial venous drainage system (Haacke et al., 2010). The pattern of iron accumulation could be considered consistent with the vascular model. The basal ganglia, midbrain, and thalamostriate regions are all drained by the medial venous drainage and all demonstrate increased iron content on MRI. The authors suggest that the iron accumulates in a pattern tracking backward through the venous outflow system.

Results from a human genetic study have been proposed to also link MS and venous malformations. A small investigation of copy number variations (CNVs) in 15 patients with MS and CCSVI did not show association between CNVs and patient phenotype, however the number of CNVs correlated with the number of stenosing malformations in the extracranial cerebrospinal veins (Ferlini et al., 2010). Although the series was small, the authors postulate that CNVs in the HLA region, a region associated with MS, may suggest a common genetic location responsible for venous formation and MS.

Multiple sclerosis commonly involves the cerebral white matter with less involvement of the spinal cord. Following the vascular model, spinal involvement may be spared due to a reflux-impeding mechanism within the dural sleeves that limit retrograde flow into radicular or perimedullary veins (Krings et al., 2006). Although no valves have been demonstrated within the spinal venous system, the venous architecture including the hairpin turn of the radicular veins has been thought to impede retrograde flow. Studies of contrast injection have demonstrated impeded retrograde opacification of the spinal cord veins.

Venous drainage is thought to play an important role in cerebral thermoregulation through countercurrent heat exchange (Zhu, 2000). At the base of the skull, both the carotid and vertebral arteries are encased by a cavernous sinus, which may in part serve as a countercurrent heat exchanger, thereby cooling the brain. In the setting of impaired venous outflow, the mechanism of counter current cooling may be impaired. For over a century, Uhthoff's phenomenon has been recognized as a temperature dependent neuronal conduction dysfunction in demyelinated fibers (Humm et al., 2004). The impaired venous outflow described in the vascular model may further compromise central nervous system temperature regulation and contribute to heat intolerance among MS patients.

\section{FINDINGS UNSUPPORTIVE OF A LINK BETWEEN CCSVI AND MS}

The initial study by Zamboni et al. (2009a) proposing the entity of CCSVI and the relationship of it to the pathogenesis of MS is based on ECD and TCCS evaluation. Basing the theory of CCSVI and MS on this measurement modality has drawn criticism due to several limitations including the ability to measure intracranial venous blood flow, operator dependence, and a lack of standardized values for diagnostic criteria (Doepp et al., 2010; Wattjes et al., 2011).

The theory of CCSVI in relation to MS holds that venous outflow is obstructed leading to venous congestion with the goal of endovascular therapy to alleviate the stenosis and thus the congestion. The proposed treatment for CCSVI is percutaneous transluminal angioplasty (PTA) as described by Zamboni et al. (2009b) in a subsequent series of endovascular treatment for CCSVI in MS patients. In their series, they defined six different malformation morphologies, including "septum/valve malformation" which indicated an anomalous valve apparatus causing flow obstruction. This was the most common type of malformation and was present 
in 30 right IJVs and 28 left IJVs. Angioplasty of a stenotic valve would seem to alleviate obstruction of flow, however it would also seem to induce valve incompetence that would result in reflux and thus retrograde flow and venous congestion. Whether this result is present was not described, and evaluation with selected angiography post-procedure and subsequent sonographic evaluation may provide clarification.

An important character of a novel scientific finding is reproducibility. Several investigators have unsuccessfully attempted to reproduce the findings of Zamboni's group. An independent group was unable to reproduce an association between venous outflow obstruction and MS using sonography (Doepp et al., 2010). This group performed transcranial color-coded sonography on 56 patients with MS and 20 control patients and found only a single patient with abnormal blood flow direction, and none of the patients fulfilled more than one criteria for CCSVI. Differences in studies including an unblinded design, a different profile of MS patients, and different sonography techniques may have contributed to the discrepancy to the findings of Zamboni et al. (2009a). A recent report using MRV to evaluate the craniocervical venous anatomy, drainage pattern, and flow, found anomalies present in MS patients and healthy controls without abnormal flow (Wattjes et al., 2011). Although limited by a small number of subjects (20 MS patients and 20 healthy controls), this also challenges the hypothesis that CCSVI is important in MS. Another study compared 21 patients with MS and 20 healthy control patients who underwent contrast enhanced MRI and found no difference regarding internal jugular venous outflow (Sundstrom et al., 2010). Additionally, early results from a study looking at prevalence of CCSVI in MS with venous Doppler identified the entity in $63 \%$ of MS patients, $26 \%$ of healthy controls, and $45 \%$ of patients with other neurological disorders (Zivadinov et al., 2010). While variability is inherently present in study designs and the technique-dependent nature of sonography, the lack of reproducibility of Zamboni's original findings remains a criticism of the hypothesis.

Radical neck dissection is performed in patients with extensive head and neck cancer and has been reported with bilateral jugular vein ligations (Ensari et al., 2008). Additionally, a small series of patients with an IJV obstruction due to various causes were studied angiographically and showed prominent channels from the sigmoidal, jugular, and vertebral systems (Cook et al., 1958). Following the vascular model of MS, this would seem to cause venous congestion resulting in the proposed inflammatory response and demyelination, but this has not been reported. An alternative explanation may be that an autoimmune predisposition or propensity to be sensitized to myelin is also needed and was not present in these patients. Furthermore, preferential cranial venous outflow may be dependent on posture, with preferential flow through the jugular system in the supine position and through the vertebral system in the erect position (Valdueza et al., 2000). If venous outflow patterns are dependent on posture in this way, then congestion from jugular obstruction may be most relevant in the supine position.

Other conditions of venous rerouting including cerebral venous thrombosis and venous embolization in treatment of vascular abnormalities such as arteriovenous malformations have not been reported to be associated with the development of MS type plaques. More recently, idiopathic intracranial hypertension (IIH) has been considered to arise from dural sinus narrowing and venous outflow obstruction. This also may result in a more restricted region of venous congestion that causes no evidence of MS type plaques due to compensation by collateral venous outflow. Additionally, venous outflow obstruction from IJV incompetence has been associated with transient global amnesia (Lewis, 1998), however this has not been associated with MS.

The venous outflow from the head may in fact be predominantly through the vertebral venous system. A postural dependence on venous outflow has been demonstrated (Valdueza et al., 2000). In healthy subjects, ultrasonography demonstrated vertebral venous outflow to be the dominant system in the erect position, while jugular venous drainage was more predominant in the supine position. The diagnostic criteria for CCSVI relies more on anterior venous outflow, which may be only partially involved in cranial venous drainage.

A comparable physiologic model may also be considered in pregnancy. Near the end of pregnancy, there is often considerable pressure on the vena cava. Venous blood is likely then shunted into the vertebral plexus, which would translate to increased cerebral venous pressure as well. Following the vascular model of pathogenesis in MS, it would seem that pregnant women exposed to extended venous outflow rerouting and possibly congestion, would develop sclerotic plaques. However, interpretation of this finding differently may in fact support the theory that the vascular model contributes to MS and that venous rerouting in pregnancy explains MS exacerbations.

\section{UNIFYING THE VASCULAR MODEL WITH CURRENT UNDERSTANDING OF MS}

The absence of a unifying theory to explain the heterogeneous disease process of MS continues to invite new hypotheses. Integration of the vascular model into the pathogenesis of MS will likely be as an adjunctive role in patients who have an underlying autoimmune susceptibility that could be enabled by venous congestion. The current understanding of the pathophysiology of MS has been an autoimmune process, however the etiology remains poorly understood. It is plausible that the vascular model offers an additional explanation to the heterogeneity of the disease and compliments the well-established role of autoimmunity. The inconsistent representation of CCSVI in the reported studies may be representative of differences in technique using a highly operator-dependent measurement tool that lacks reference values in small non-randomized patient populations with differing disease profiles. The vascular model demands the presence of multiple factors to allow for a role in the disease process. It is possible that a subset of MS patients harbor several specific characteristics. Unifying the current understanding of MS pathogenesis with the hypothesis of a vascular model may be best explained with the patient meeting several criteria including a propensity to be sensitized to myelin, and a venous drainage architecture that results in congestion. 


\section{DIAGNOSIS}

If indeed a link between venous outflow obstruction and MS is established, diagnosis will likely remain challenging. The cerebrospinal venous outflow is complex and dynamic making evaluation with any single modality challenging. Sonography is heavily operator dependent with no standardized criteria, MR flow quantification has not been validated (Wattjes et al., 2011), and catheter angiography is largely limited to supine studies neglecting the potential role of postural dependence. The considerable limitations to transcranial Doppler evaluation include velocity measurement and insonation angle as well as operator variability. The IJV is a thin-walled compressible structure susceptible to changes from sonography probe pressure and adjacent structures. The extracranial venous system is susceptible to morphology changes resulting from hydration, body position, compression, and intrathoracic pressure changes. Furthermore, standard values for venous sonography will have to be established.

Although a multitude of magnetic resonance imaging sequences exist, MRV is inferior to Doppler sonography and selective venography in identifying cervical venous abnormalities (Hojnacki et al., 2010). Limitations of MRV are likely related to the static nature of the modality as well as the morphological venous changes due to body position and compression.

Catheter venogram remains the gold standard for venous system evaluation, however no reference data are available. Additionally, the postural dependence of the venous outflow system may introduce an additional challenge in accurate evaluation.

\section{TREATMENT}

A discussion on treatment may be premature, however it should be noted that if indeed a link is demonstrated, considerable challenges must be overcome to optimize treatment. In the prospective endovascular treatment pilot trial by Zamboni et al. (2009a) PTA was performed on 65 patients and 18-month follow-up was described. They reported improvement in venous pressure, MS clinical outcome measures, and quality of life assessments (Zamboni et al., 2009b). This was an unblinded pilot series showing that endovascular therapy is feasible with low complications, however this study was small, had no control subjects, and demonstrated a restenosis rate of $47 \%$. Although complications were minimal in this initial study, a subsequent report of intracranial hemorrhage was reported as well as a case with stent migration requiring open-heart surgery (Qui, 2010).

Current device technology and interventional skill is available to afford endovascular remodeling, however further understanding of the pathophysiology is needed. The overall restenosis rate of $47 \%$ suggests balloon angioplasty may be insufficient and that stent implantation may be necessary for durable revascularization. Balloon angioplasty and stent implantation in the arterial system has been well-described and utilized for many years with an adequate understanding of feasibility, safety, and long-term effects. Unlike the arterial system, the venous system lacks a supportive multilayered architecture, theoretically posing a greater risk of vessel rupture. Additionally, the venous wall characteristics leading to the described obstructions are not well understood and may involve hyperplasia, webbing, trabeculation, or fenestration and therefore respond differently to varying treatment modalities. The long-term effects of endoluminal scaffold implantation with stents in the venous system is not as well understood and will need further investigation of host response, such as endothelialization, and the appropriate subsequent antithrombotic regimen. Stent design will also likely be important in achieving safety, durability, and efficacy, however this will only follow a better understanding of the lesion pathology. Although endovascular technique and device technology are available to implement invasive treatment, data on safety and efficacy are lacking.

\section{FUTURE}

Grants for research investigating CCSVI have thus far been awarded for seven projects by the National MS Society and the MS Society of Canada (FAQs About New CCSVI Grants, 2011). Intentional cerebral venous obstruction in an animal model of MS could also further elucidate the role of a vascular model in MS by testing the hypothesis that venous congestion leads to BBB disruption and enables the autoimmune response. Additionally, review of MS registries for an association between IIH and severity of MS disease may also be helpful in better understanding the role of venous obstruction.

\section{SUMMARY}

The association between MS and venous system pathology is compelling given the perivenule topography of plaques that has been demonstrated both histologically and with MR imaging, however the fundamental mechanism of venous dysfunction is less clear. Venous outflow obstruction leading to venous congestion and BBB dysfunction is conceivable, however the redundancy of the venous anatomy would seem to demand venous obstruction at numerous places to exhaust collateral pathways. Explaining MS by a vascular model is further challenged by an incomplete understanding of venous system physiology, which is highlighted by the postural dependence of venous outflow. The vascular model may relate to only a portion of those patients with MS, in which particular patient selection would be needed to demonstrate reproducibility.

\section{CONCLUSION}

The incomplete understanding of the pathophysiology and effective treatment in a chronic and debilitating disease that affects up to 2.5 million people worldwide is the foundation of the enthusiasm for a proposed treatable vascular model such as CCSVI. If confirmed, the vascular model would change understanding of the pathophysiology of MS considerably. The proposal that CCSVI underlies the pathophysiology of MS has emerged with several compelling reports, however a careful understanding of several elements is needed. A better understanding of the pathophysiology, standardized diagnostic criteria, and rigorous randomized evaluation of intervention with long-term outcomes are needed before widespread adoption of endovascular therapies. Further endovascular technique and device developments will then be needed to ensure durable efficacy. Endovascular therapy for MS remains investigational and should remain limited to multicenter, blinded, randomized, controlled clinical trials. 


\section{REFERENCES}

Adams, C. W. (1988). Perivascular iron deposition and other vascular damage in multiple sclerosis. J. Neurol. Neurosurg. Psychiatr. 51, 260-265.

Anderson, D. W., Ellenberg, J. H., Leventhal, C. M., Reingold, S. C., Rodriguez, M., and Silberberg, D. H. (1992). Revised estimate of the prevalence of multiple sclerosis in the United States. Ann. Neurol. 31, 333-336.

Barnett, M. H., and Sutton, I. (2006). The pathology of multiple sclerosis: a paradigm shift. Curr. Opin. Neurol. 19, 242-247.

Braun, J. P., and Tournade, A. (1977). Venous drainage in the craniocervical region. Neuroradiology 13, 155-158.

Chou, C. H., Chao, A. C., and Hu, H. H. (2002). Ultrasonographic evaluation of vertebral venous valves. AJNR Am. J. Neuroradiol. 23, 1418-1420.

Cook, A. W., Freund, H. R., and Browder, E. J. (1958). Venous patterns following occlusion of the jugular system as demonstrated by jugular venography. Surgery 44, 338-344.

Doepp, F., Paul, F., Valdueza, J. M. Schmierer, K., and Schreiber, S. J. (2010). No cerebrocervical venous congestion in patients with multiple sclerosis. Ann. Neurol. 68, 173-183.

Eckenhoff, J. E. (1970). The physiologic significance of the vertebral venous plexus. Surg. Gynecol. Obstet. 131, 72-78.

Ensari, S., Kaptanoglu, E., Tun, K., Gün, T., Beskonakli, E., Celikkanat, S., Dere, H., and Cekirge, S. (2008). Venous outflow of the brain after bilateral complete jugular ligation. Turk. Neurosurg. 18, 56-60.

FAQs About New CCSVI Grants. (2011). Available at: http://www. nationalmssociety.org/research/ intriguing-leads-on-the-horizon/ ccsvi/faqs-about-new-ccsvi-grants/ index.aspx [accessed April 25, 2011].

Ferlini, A., Bovolenta, M., Neri, M., Gualandi, F., Balboni, A., Yuryev, A., Salvi, F., Gemmati, D., Liboni, A., and Zamboni, P. (2010). Custom CGH array profiling of copy number variations (CNVs) on chromosome 6p21.32 (HLA locus) in patients with venous malformations associated with multiple sclerosis. BMC Med. Genet. 11, 64. doi: 10.1186/1471-2350-11-64

Fog, T. (1965). The topography of plaques in multiple sclerosis with special reference to cerebral plaques.
Acta Neurol. Scand. Suppl. 15, 1-161.

Haacke, E. M., Garbern, J., Miao, Y., Habib, C., and Liu, M. (2010). Iron stores and cerebral veins in MS studied by susceptibility weighted imaging. Int. Angiol. 29, 149-157.

Hojnacki, D., Zamboni, P., LopezSoriano, A., Galleotti, R., Menegatti, E., Weinstock-Guttman, B., Schirda, C., Magnano, C., Malagoni, A. M., Kennedy, C., Bartolomei, I., Salvi, F., and Zivadinov, R. (2010). Use of neck magnetic resonance venography, Doppler sonography and selective venography for diagnosis of chronic cerebrospinal venous insufficiency: a pilot study in multiple sclerosis patients and healthy controls. Int. Angiol. 29, 127-139.

Humm, A. M., Beer, S., Kool, J., Magistris, M. R., Kesselring, J., and Rosler, K. M. (2004). Quantification of Uhthoff's phenomenon in multiple sclerosis: a magnetic stimulation study. Clin. Neurophysiol. 115, 2493-2501.

Hurst, R. W. (2008). Interventional Neuroradiology. New York, NY: Informa Healthcare.

Ibukuro, K., Fukuda, H., Mori, K. and Inoue, Y. (2001). Topographic anatomy of the vertebral venous system in the thoracic inlet. AJR Am. J. Roentgenol. 176, 1059-1065.

Koch-Henriksen, N., and Sorensen, P. S. (2010). The changing demographic pattern of multiple sclerosis epidemiology. Lancet Neurol. 9, 520-532.

Krings, T., Mull, M., Bostroem, A., Otto, J., Hans, F. J., and Thron, A. (2006). Spinal epidural arteriovenous fistula with perimedullary drainage. Case report and pathomechanical considerations. J. Neurosurg. Spine 5, 353-358.

Lee, A. B., Laredo, J., and Neville, R. (2010). Embryological background of truncular venous malformation in the extracranial venous pathways as the cause of chronic cerebro spinal venous insufficiency. Int. Angiol. 29, 95-108.

Lewis, S. L. (1998). Aetiology of transient global amnesia. Lancet 352, 397-399.

Putnam, T. J. (1935). Studies in multiple sclerosis; "encephalitis" and sclerotic plaques produced by venular obstruction. Arch. Neurol. Psychiatry 33, 929-940.

Qui, J. (2010). Venous abnormalities and multiple sclerosis: another breakthrough claim? Lancet Neurol. 9, 464-465.
San Millan Ruiz, D., Gailloud, P., Rufenacht, D. A., Delavelle, J., Henry, F., and Fasel, J. H. (2002). The craniocervical venous system in relation to cerebral venous drainage. AJNR Am. J. Neuroradiol. 23, 1500-1508.

Sanchez-Hanke, M., Puschel, K., and Leuwer, R. (2000). Anatomy of the valve system of the internal jugular vein. Laryngorhinootologie 79 , 332-336.

Silva, M. A., Deen, K. I., Fernando, D. J., and Sheriffdeen, A. H. (2002). The internal jugular vein valve may have a significant role in the prevention of venous reflux: evidence from live and cadaveric human subjects. Clin. Physiol. Funct. Imaging 22, 202-205.

Steinke, K., and Moghaddam, A. (2009). Azygos arch valves at computed tomography angiography and pitfalls related to its variety in appearance and function. J. Comput. Assist. Tomogr. 33, 721-724.

Sundstrom, P., Wahlin, A., Ambarki, K. Birgander, R., Eklund, A., and Malm, J. (2010). Venous and cerebrospinal fluid flow in multiple sclerosis: a case-control study. Ann. Neurol. 68, 255-259.

Talbert, D. G. (2008). Raised venous pressure as a factor in multiple sclerosis. Med. Hypotheses 70, 1112-1117.

Tan, I. L., van Schijndel, R. A., Pouwels, P. J., van Walderveen, M. A., Reichenbach, J. R., Manoliu, R. A., and Barkhof, F. (2000). MR venography of multiple sclerosis. AJNR Am. J. Neuroradiol. 21, 1039-1042.

Valdueza, J. M., von Munster, T., Hoffman, O., Schreiber, S., and Einhaupl, K. M. (2000). Postural dependency of the cerebral venous outflow. Lancet 355, 200-201.

Wattjes, M. P., van Oosten, B. W., de Graaf, W. L., Seewann, A., Bot, J. C., van den Berg, R., Uitdehaag, B. M., Polman, C. H., and Barkhof, F. (2011). No association of abnormal cranial venous drainage with multiple sclerosis: a magnetic resonance venography and flow-quantification study. J. Neurol. Neurosurg. Psychiatr. 82, 429-435.

Yeh, B. M., Coakley, F. V., Sanchez, H. C., Wilson, M. W., Reddy, G. P., and Gotway, M. B. (2004). Azygos arch valves: prevalence and appearance at contrast-enhanced CT. Radiology 230, 111-115.

Zamboni, P., Galeotti, R., Menegatti, E., Malagoni, A. M., Tacconi, G., Dall'Ara, S., Bartolomei, I., and Salvi, F. (2009a). Chronic cerebrospinal venous insufficiency in patients with multiple sclerosis. J. Neurol. Neurosurg. Psychiatr. 80, 392-399.

Zamboni, P., Galeotti, R., Menegatti, E., Malagoni, A. M., Gianesini, S., Bartolomei, I., Mascoli, F., and Salvi, F. (2009b). A prospective open-label study of endovascular treatment of chronic cerebrospinal venous insufficiency. J. Vasc. Surg. 50, 13481358.e1-3.

Zamboni, P., Menegatti, E., Bartolomei, I., Galeotti, R., Malagoni, A. M., Tacconi, G., and Salvi, F. (2007). Intracranial venous haemodynamics in multiple sclerosis. Curr. Neurovasc. Res. 4, 252-258.

Zhu, L. (2000). Theoretical evaluation of contributions of heat conduction and countercurrent heat exchange in selective brain cooling in humans. Ann. Biomed. Eng. 28, 269-277.

Zivadinov, R., Marr, K., Ramanathan, M., Zamboni, P., Benedict, R. R. H B., Cutter, G., Kennedy, C., Elfadil, M., Hojnacki, D., Munschauer, F., Reuther, J., Brooks, C., Hunt, K., Andrews, M., and WeinstockGuttman, B. (2010). Combined transcranial and extracranial venous doppler evaluation (CTEVD). Description of the design and interim results of an epidemiological study of the prevalence of chronic cerebrospinal venous insufficiency in MS and related diseases. Neurology 74, A545.

Conflict of Interest Statement: The authors declare that the research was conducted in the absence of any commercial or financial relationships that could be construed as a potential conflict of interest.

Received: 03 May 2011; accepted: 28 June 2011; published online: 14 July 2011. Citation: Lazzaro MA, Zaidat OO, Mueller-Kronast N, Taqi MA and Woo $D$ (2011) Endovascular therapy for chronic cerebrospinal venous insufficiency in multiple sclerosis. Front. Neur. 2:44. doi: 10.3389/fneur.2011.00044

This article was submitted to Frontiers in Endovascular and Interventional Neurology, a specialty of Frontiers in Neurology. Copyright (c) 2011 Lazzaro, Zaidat, Mueller-Kronast, Taqi and Woo. This is an open-access article subject to a nonexclusive license between the authors and Frontiers Media SA, which permits use, distribution and reproduction in other forums, provided the original authors and source are credited and other Frontiers conditions are complied with. 